\title{
Un diagnóstico de la universidad mexicana en la obra EI libro de la universidad imaginada
}

\author{
A diagnosis of the mexican university in the book: The book of the imagined \\ university
}

\begin{abstract}
Um diagnóstico da universidade mexicana no livro: $O$ livro da universidade imaginada

Jenaro Reynoso Jaime

Universidad Autónoma del Estado de México, México

rjenaro@hotmail.com

http://orcid.org/0000-0003-1807-0317
\end{abstract}

\section{Resumen}

Una de las tendencias en los estudios dedicados a instituciones educativas como la universidad sostiene que la práctica investigativa tiene sentido si propone soluciones a los problemas detectados. Para este enfoque de investigación, la intervención en el funcionamiento de la institución solo puede realizarse cuando se hace sobre la base de las conclusiones que arrojan los estudios acerca de las condiciones presentes en las que aquellas funcionan; a su vez, la compresión y valoración que se hace del modo en que opera una institución solo puede lograrse después de revisar retrospectivamente el proceso que la llevó al estado en que se diagnostica.

De esa manera, la imaginación y elaboración de propuestas de solución a problemas detectados, en un aspecto o en el conjunto de la universidad, es procedente cuando quienes la ejercitan lo hacen con los vastos saberes que la formación, el estudio y la experiencia profesional les proporcionan. Tal es el caso de Eduardo Ibarra Colado, quien a partir de su experiencia académica ha promovido la escritura a ocho manos de El libro de la universidad imaginada. Hacia una universidad situada entre el buen lugar y ningún lugar, en el cual la colectividad autoral presenta a la universidad mexicana como una institución inoperante y vieja que debe cambiar hacia una propuesta que aparentemente parece utópica, pero cuyos argumentos llevan a pensarla como necesaria. Por ello, los objetivos del presente documento 

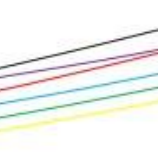

\section{Revista Iberoamericana para la Investigación y el Desarrollo Educativo ISSN $2007-7467$}

son ofrecer un acercamiento al concepto diagnóstico, describir la trayectoria del iniciador de la idea plasmada en el referido libro y presentar los rasgos de la universidad anquilosada, la cual, según los autores, debe desaparecer.

Palabras clave: diagnóstico, historia, trayectoria académica, universidad, utopía.

\section{Abstract}

One of the trends in studies devoted to educational institutions, such as the university, argues that research practice makes sense if it proposes solutions to the problems detected. For this research approach, the intervention in the operation of the institution can only be carried out when it is done on the basis of the conclusion drawn by the studies about the present conditions in which they work; in turn, the compression and assessment that is made of the way in which an institution operates can only be achieved after retrospectively reviewing the process that led to the state I which it is diagnosed.

In this way, the imagination and development of solutions to problems detected, in one aspect or in the whole of the university, is appropriate when those who exercise it do so with the vast knowledge that training, study and professional experience they provide. Such is the case of Eduardo Ibarra Colado, who, based on his academic experience, promotes eight-hand writing of the text: The book of the imagined university. Towards a university located between the good place and nowhere, in which the authorial community diagnoses the Mexican university as an inoperative and unnecessary institution that needs to be changed by a proposal that apparently seems utopian; but the arguments lead to think of it as necessary. In this text an approach to the diagnostic concept is made, the trajectory of the initiator of the idea is described and the features of the stagnant university that should disappear are presented.

Keywords: diagnosis, history, academic trajectory, university, utopia. 

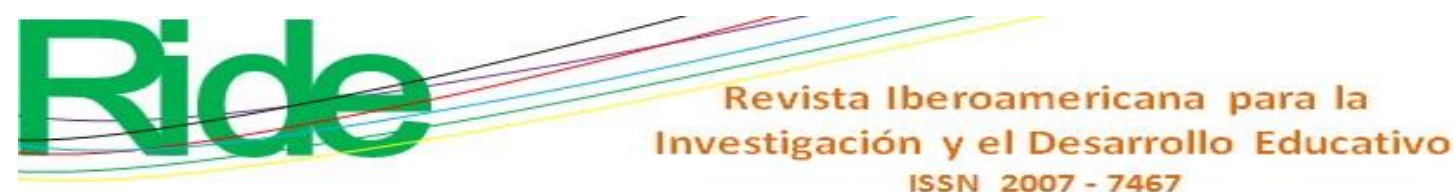

\section{Resumo}

Uma das tendências em estudos dedicados a instituições de ensino, como a universidade, é que a prática de pesquisa faz sentido se propõe soluções para os problemas detectados. Para esta abordagem de pesquiza, a intervenção no funcionamento da instituição só pode ser realizada quando é feita com base na conclusão tirada pelos estudos sobre as condições atuais em que trabalham; por sua vez, a compressão e a avaliação feitas da maneira pela qual uma instituição opera só podem ser alcançadas depois de revisar retrospectivamente o processo que levou ao estado que é diagnosticado.

Desta forma, a imaginação e o desenvolvimento de soluções para os problemas detectados, em um aspecto ou em toda a universidade, é apropriado quando aqueles que o exercem o fazem com o vasto conhecimento que a formação, o estudo e a experiência profissional proporcionam. É o caso de Eduardo Ibarra Colado, que, baseado em sua experiência acadêmica, promove a redação de oito letras do texto O livro da universidade imaginada. Para uma universidade localizada entre o lugar bom e o lugar nenhum, em que a comunidade autoral diagnostica a universidade mexicana como uma instituição inoperante e antiga que precisa ser mudada por uma proposta que parece aparentemente utópica; mas cujos argumentos nos levam a pensar nisso como necessário. Neste texto é feita uma abordagem do conceito de diagnóstico, é descrita a trajetória do iniciador da ideia e apresentadas as características da universidade estagnada que deve desaparecer.

Palavras-chave: diagnóstico, história, trajetória académica, universidade, utopia.

Fecha Recepción: Agosto 2018

Fecha Aceptación: Enero 2019 


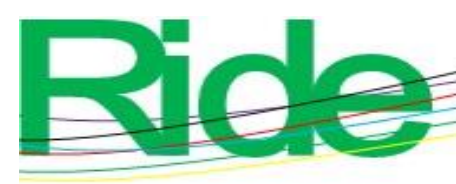

Revista Iberoamericana para la

Investigación y el Desarrollo Educativo

ISSN 2007 - 7467

\section{Introducción}

En el presente artículo se presenta una exégesis o análisis hermenéutico de El libro de la universidad imaginada. Hacia una universidad situada entre el buen lugar y ningún lugar, cuya primera versión fue resultado de la mente inquieta de Eduardo Ibarra Colado (Buendía, 2014) y de la mente un tanto obsesiva de Luis Porter Galetar; a esta luego se sumaron seis dialogantes ${ }^{1}$, quienes posteriormente fueron reescribiéndola, de ahí que se pueda asegurar que la versión definitiva es un texto construido colectivamente, publicado en 2012 por la Universidad Autónoma Metropolitana Cuajimalpa y Juan Pablo Editor.

Con la lectura que se presenta a continuación se intentan comprender los argumentos históricos de los autores sobre los diferentes aspectos de la universidad para calificarla actualmente como una institución en crisis que debe ser transformada tomando como base la valoración de sus modificaciones recientes. Para lograr este objetivo se plantearon las siguientes preguntas: ¿cuál es el proceso histórico que, según los autores, ha seguido la universidad mexicana hasta su situación actual?, ¿cuáles han sido las variables (internas, nacionales o internacionales) que han provocado la situación que vive la universidad?, y ¿cuáles características tienen las diferentes actividades de la universidad?

Las breves respuestas que en este documento se ofrecen están compuestas, en principio, por una definición de la frase diagnóstico histórico, la cual sirve de apoyo para centrar el análisis de la referida obra; luego, con base en el recorrido intelectual de Eduardo Ibarra, se profundiza en el funcionamiento de la universidad de fines del siglo XX y principios del XXI. Posteriormente, en el tercer apartado se destacan los aspectos en los cuales los autores coinciden en relación con la siguiente premisa: la universidad como institución excluyente, petrificada, desinstitucionalizada, sin vida e innecesaria socialmente, la cual se encuentra destinada a ser sustituida con una nueva utopía, cuyas características se discuten en otro texto.

\footnotetext{
${ }^{1}$ Los seis dialogantes fueron Lilian Álvarez, Daniel Cazés, Raquel Glazman, Arturo Guillaumin, Javier Ortiz y Lourdes Pacheco.
} 


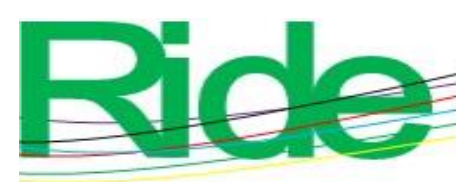

Revista Iberoamericana para la

Investigación y el Desarrollo Educativo

ISSN 2007 - 7467

\section{Método}

El presente escrito fue el resultado de un proceso de investigación en el que se aplicó el método filosófico de la hermenéutica, entendida esta como la disciplina de la interpretación de textos. Este ejercicio tuvo como base los postulados que Hans-George Gadamer expone en su obra Verdad y método, entre los cuales destaca la idea de que la interpretación es una forma de conocimiento que trasciende la explicación científica moderna, ya que implica un proceso más complejo en el que se enfrentan el horizonte cultural (presente en el lector) con la historia y la cultura del autor del texto en su contexto (Gadamer, 1975).

Esto significa que el nuevo conocimiento o su interpretación surgen de la fusión de los horizontes del autor del texto (implícito en el texto) y del lector que busca comprender al otro a través de su carga cultural y su predisposición. En este caso concreto, el lector-autor del presente artículo (desde su formación profesional como historiador) busca saber por qué el principal autor de El libro de la universidad imaginada califica a la institución educativa como decrépita e incongruente con los fines para los que fue creada. Con esta idea, se procede a investigar la trayectoria académica de Ibarra Colado para intentar explicar el origen de sus argumentos.

\section{Elementos para una definición de diagnóstico histórico}

Según el Diccionario de la Real Academia Española, el diagnóstico es la calificación que da el médico a la naturaleza de una enfermedad después de observar sus síntomas y signos. Esta acción solo se puede desprender del contraste sistematizado del conocimiento acumulado acerca del funcionamiento normal o ideal del organismo y los síntomas que experimenta con dolor o sufrimiento el ser humano, los cuales se comprueban con la observación, la auscultación física y los estudios clínicos. De manera análoga, diagnosticar a las instituciones consiste en aplicar métodos para reconocer los cambios que en su estructura y funciones han operado a partir del ideal establecido en su normatividad, en la filosofía que encierran sus propósitos originales y en el papel que los discursos de las fuerzas sociales le asignan históricamente. 

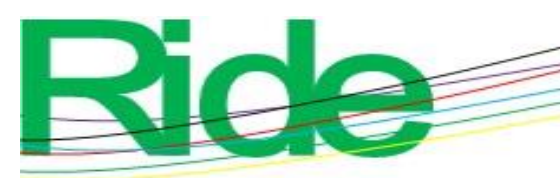

\section{Revista Iberoamericana para la Investigación y el Desarrollo Educativo ISSN $2007-7467$}

Un diagnóstico histórico de la universidad, por ende, implica no solo la comparación entre el deber ser y el ser, sino también el reconocimiento del ser con base en el saber acumulado por el sujeto que enuncia el síntoma y caracteriza la falla en la acción que se esperaba. Entre los saberes necesarios para el diagnóstico institucional se encuentran, fundamentalmente, la conciencia histórica del tiempo que se vive; esto es, haber comprendido el contexto específico en el que la institución educativa se desempeña, lo cual incluye el reconocimiento de distintas fuerzas (como el Estado, los empresarios o los movimientos sociales) que son capaces de promover o imponer cambios a la institución universitaria. De esa manera, el diagnóstico interrelaciona el presente y el pasado no solo porque la intención de valorar el estado de una institución se hace desde un aquí y un ahora, sino porque ese aquí y ahora emerge del proceso llamado historia, en el cual metodológicamente se debe hacer un recorrido inverso para encontrar las causales de las variables observadas desde el presente.

El conocimiento producido por los estudios institucionales aporta teorías con las que es posible visualizar aquellos aspectos determinantes de una problemática institucional; con la aplicación de aquellas y el uso de las técnicas y los instrumentos adecuados, un especialista puede conseguir un diagnóstico más o menos certero para presentarlo a quien toma decisiones; sin embargo, un diagnóstico histórico es más exhaustivo porque procura, por un lado, conocer las raíces de una situación con el propósito de hallar la multicausalidad de los hechos; por otro lado, este tipo de exploración resulta útil porque permite comprender de mejor manera el fenómeno indagado, especialmente cuando el estudioso es contemporáneo del proceso histórico de la institución, pues no solo comparte las permanencias y los cambios que hacen a la universidad, sino que también ha sido parte de ella. El diagnóstico histórico, además, brinda una más amplia comprensión de los hechos si los sujetos realizan acercamientos y reflexiones parciales y periódicas, lo que resulta vital para construir una visión histórica de largo plazo.

Al respecto, vale destacar que una visión de este tipo no concluye con asociar a los hechos institucionales presentes una explicación de los determinantes del pasado reciente o lejano ni con sufrir o disfrutar de forma alterna las transformaciones de la institución como 

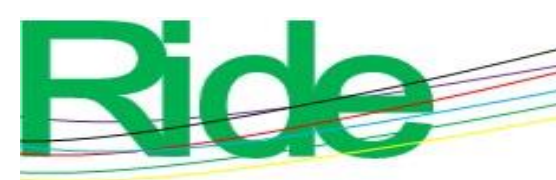

\section{Revista Iberoamericana para la Investigación y el Desarrollo Educativo ISSN $2007-7467$}

espacio de carácter laboral y, por tanto, vivencial, pues también exige una profunda reflexión para acumular conocimiento a través de acercamientos periódicos en los que se combine la delimitación de aspectos problemáticos desde posturas teóricas que permitan lograr la comprensión hermenéutica. De esta forma, el diagnóstico histórico se convierte en una plataforma desde la cual se pueden ir formulando paulatinamente distintos tipos de soluciones al funcionamiento institucional, las cuales se pueden presentar como cambios superficiales, como reformas de tipo estructural o como formulación de utopías, como lo hacen los autores del libro de la universidad imaginada.

\section{Práctica, teoría e investigación para diagnosticar la universidad: el caso de Eduardo Ibarra Colado}

Una vida que encarnó las condiciones desde las cuales es posible diagnosticar el funcionamiento de la universidad fue la de Eduardo Ibarra Colado, quien nació el 25 de agosto de 1957 en el Distrito Federal, hoy Ciudad de México. En ese espacio geográfico obtuvo su formación básica escolar y profesional: terminó la licenciatura en Administración hacia 1980 en la Universidad Autónoma Metropolitana-Xochimilco (UAM); obtuvo en 1983 la maestría en Administración Pública en el Centro de Investigación y Docencia Económica (CIDE) y en 1999 el doctorado en Sociología con la tesis La universidad en México hoy: gubernamentalidad y modernización, un estudio profundo de la institución universitaria en la Facultad de Ciencias Políticas y Sociales de la Universidad Nacional Autónoma de México (S/A, S/F, Resumen).

Ingresó como profesor temporal de tiempo completo con la categoría más baja (asistente A) en el Departamento de Economía de la Universidad Autónoma Metropolitana Iztapalapa, unidad de la UAM, donde desarrolló una carrera como académico hasta 2005, cuando contribuyó con su presencia en la fundación de la Unidad Cuajimalpa de la misma casa de estudios. En esta permaneció hasta 2011 como jefe del Departamento de Estudios Institucionales. Luego, en 2012 cambió su adscripción al Departamento de Producción Económica de la UAM-Xochimilco, de donde había egresado en 1980. En las tres unidades de la UAM donde Ibarra Colado hizo su vida laboral cumplió con actividades de docencia en diferentes niveles educativos, realizó investigación y promovió la formación de grupos para 

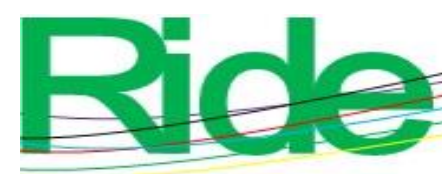

Revista lberoamericana para la

Investigación y el Desarrollo Educativo

ISSN $2007-7467$

estudiar a la universidad. Además, fue impulsor de programas de posgrado y difusor del conocimiento en diferentes foros nacionales y extranjeros.

En su carácter de académico mexicano, perteneciente al sistema nacional de investigadores, se dedicó al área de conocimiento conocida como sociología de las instituciones, dentro de la cual cultivó las especialidades de gestión y organización de las instituciones y estudios sobre la universidad; esto lo convirtió en un verdadero científico de la universidad, lo cual fue reconocido con su ingreso a la Academia Mexicana de las Ciencias en 2001. Además, fue condecorado con el Premio a la Investigación 2003 por parte de la UAM y consiguió otros reconocimientos, entre los que se destacan los siguientes:

La nominación de su trabajo Entrepreneurialization of the university: Shadows of the US History on the ongoing mexican reform entre las cinco mejores ponencias de la Critical Management Studies Division en el Congreso de la Academy of Management (Philadelphia 2007) y el reconocimiento de su trabajo Organization Studies and Epistemic coloniaty in America Latina: thinking otherness from the margins como uno de los artículos más relevantes publicados por SAGE al ser seleccionado para integrar la obra SAGE Works in organization studies (Thousand Oaks/ Londres/ Nueva Dheli, 2009). Además recibió el Literati Network Award for Excelence 2011 por el mejor artículo del año publicado en la revista Critical Perspectives on International Bussines (Emerald en coautoría con Alex Faria y Ana Guedes de la Fundación Getulio Vargas de Brasil) (Ibarra, S/A, p. 7).

Estos reconocimientos fueron el momento culminante de una carrera en la universidad que le permitió conocerla no solo mediante la práctica de la docencia, sino también a través de la reflexión y la investigación desde la perspectiva de los estudios institucionales; en ese sentido, fueron importantes los grupos de investigación en los que participó, los proyectos que impulsó, sus publicaciones sobre el tema de la universidad y las actividades de actualización que realizó a lo largo de su vida (S/A, S/F, Publicaciones).

Respecto a la fundación de grupos, entre 1983 y 1984 agrupó a diez investigadores para analizar la teoría anglosajona e iniciar estudios empíricos sobre el tema institucional en 

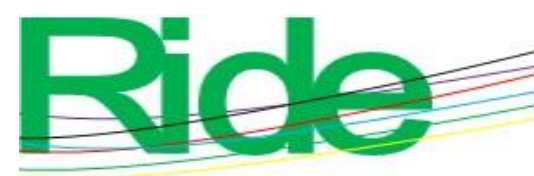

Revista Iberoamericana para la

Investigación y el Desarrollo Educativo

ISSN $2007-7467$

la economía y la sociedad mexicana con base en las tres siguientes líneas de investigación: a) individuo, trabajo y organización; b) estructuras, decisiones y diseño organizacional, y c) modelos organizacionales y contextos internacionales. Entre los resultados que tuvo este grupo estuvieron la creación de un área de estudios institucionales (antes inexistente en la universidad mexicana), la fundación de la maestría y el doctorado en estudios institucionales, varias publicaciones sobre el tema y la obtención del Premio a las Áreas de Investigación en 1993 (Ibarra, S/A).

Otras iniciativas con resultados similares fueron la creación del Programa de Investigaciones sobre Educación Superior en México (entre 2001 y 2004), la coordinación del Programa de Investigaciones la Educación Superior Pública en México (entre 2004 y 2008) y la fundación del Departamento de Estudios Institucionales de la UAM-Cuajimalpa junto con el cuerpo académico Estudios institucionales, gestión pública y desarrollo social, que sostendría el funcionamiento de la nueva casa de estudios mediante el desarrollo de cuatro programas de investigación: a) estudios institucionales en economía: instituciones, desarrollo económico y acción estatal; b) organizaciones basadas en el conocimiento: valor social y nuevos agentes y configuraciones; c) instituciones, organizaciones y acción pública para el desarrollo social, y d) análisis institucional y jurídico en México (Ibarra, S/A).

En un plano más concreto, los proyectos de investigación que Ibarra desarrolló también dan cuenta de la profundidad que alcanzó en el conocimiento de la universidad para diagnosticar su desempeño histórico a inicios del siglo XXI. Comenzó entre 1984 y 1985 con el diseñó y coordinación de cinco investigadores para desarrollar el proyecto Problemas y posibilidades en el estudio de las organizaciones en México con el propósito de detectar los problemas para fundamentar teóricamente el estudio de realidades como la mexicana.

El resultado fue la publicación del libro Ensayos críticos para el estudio de las organizaciones en México. Entre 1986 y 1987 profundizó sobre la temática a través de la coordinación del proyecto colectivo Ensayos metodológicos para el estudio de las instituciones, en el que los autores examinaron los paradigmas, los aportes relevantes de las disciplinas sociales y las estrategias consolidadas para el estudio de las organizaciones; de 
este proyecto también surgió el libro El orden organizacional: poder, estrategia y contradicción (Ibarra, S/A).

Desde 1988 hasta 1990, Ibarra formuló un nuevo proyecto titulado Análisis estratégico de las organizaciones, e invitó a varios investigadores a reflexionar en torno a la vinculación organización-sociedad en los casos de organizaciones como la empresa, la burocracia, el ejército y la educación superior a partir del concepto de estrategia. Del seminario colectivo resultó la primera parte, relacionada con los estudios organizacionales, del libro Desarrollo, estrategia y política económica. Entre 1990 y 1991 integró un grupo que organizaría el Primer Coloquio Internacional de Estudios Organizacionales como parte del proyecto Modelos organizacionales para América Latina, el cual convocó a investigadores de Argentina, Brasil, Canadá, Colombia, Chile, Japón, Perú, Reino Unido, Venezuela y México para estudiar el impacto de la globalización, la regionalización, la tecnología y las transformaciones del Estado en las organizaciones. Los trabajos del coloquio integraron el libro Modelos organizacionales para América Latina, el cual no se publicó por restricciones presupuestarias (Ibarra, S/A).

Al respecto, vale destacar que con el proyecto sobre el análisis de las organizaciones se dio un primer acercamiento a la organización de la educación superior; sin embargo, fue con el proyecto Educación superior y análisis organizacional de 1990-1992 cuando se propuso "analizar la problemática de la modernización de la educación superior y la ciencia en México, confrontándola con la experiencia estadounidense"; con esta iniciativa se buscaban evaluar los cambios en el sistema y en las instituciones, lo cual incluía lo siguiente:

Abordar el análisis de las políticas estatales en la materia, destacando sus impactos más específicos e inmediatos, como el de realidades institucionales que muestran de manera ejemplar los procesos más específicos de conformación y cambio (se consideran como casos relevantes los recientes procesos de cambio institucional experimentados por la UAM y el CONACYT) (Ibarra, S/A, p. 52). 

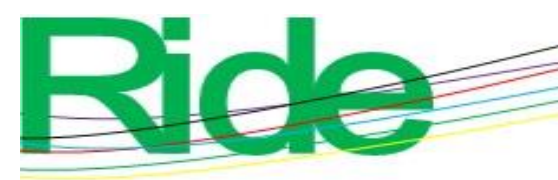

\section{Revista Iberoamericana para la Investigación y el Desarrollo Educativo ISSN 2007 - 7467}

Luego, entre abril de 1990 y octubre de 1992 se realizaron sesiones de seminario para discutir colectivamente los avances de la investigación acerca de los procesos de modernización de la universidad; al año siguiente fue publicado el libro La universidad ante el espejo de la excelencia. Enjuegos organizacionales, coordinado por Eduardo Ibarra Colado, el cual refleja la aplicación directa de la teoría cultivada durante casi diez años a la organización universitaria y al sistema de producción científica en México.

De forma simultánea, impulsó el proyecto Desarrollo de la administración en la $U A M$ con otros once investigadores para estudiar las posibilidades de diseñar e implementar el primer programa de maestría y doctorado en estudios organizacionales en el país. De este proyecto surgieron documentos que sirvieron como base para futuras decisiones: se elaboró la propuesta de desarrollo de la disciplina de administración en la UAM-Iztapalapa, el anteproyecto para la creación de una revista especializada en estudios organizacionales y los planes de estudio de maestría y doctorado en Estudios Institucionales, que comenzaron a operar en septiembre de 1995 (Ibarra, S/A).

El proceso de vinculación de la teoría organizacional con la experiencia de la institución universitaria tuvo su siguiente fase en el proyecto Educación superior y teoría de la organización llevado a cabo entre 1994 y 1996. En esta nueva iniciativa, Ibarra coordinó a investigadores de la talla de Cynthia Hardy (de Canadá), Herhard Friedberg y Christine Musselin (de Francia), Jane Marceau (de Australia) y Arturo Castillo (del CIDE), con la intención de aplicar la teoría de la organización al estudio de la educación superior con base en cuatro líneas de investigación:

1) Aportes y limitaciones de la teoría de la organización para estudiar la educación superior en México; 2) el análisis estratégico y los estudios comparativos a nivel internacional a partir de los aportes realizados en Francia; 3) las propuestas desarrolladas recientemente para analizar las decisiones estratégicas y los estilos de liderazgo en las universidades tomando como punto de referencia el caso brasileño y; 4) los nuevos enfoques centrados en el análisis de los cambios en las formas de organización y su impacto en las universidades a partir de la conformación de redes interorganizacionales 
entre agencias gubernamentales, empresas y universidades y centros de investigación (Ibarra, S/A, p. 51).

Posteriormente, desde 1995 hasta el año 2000 consolidó e internacionalizó los estudios organizacionales a través de proyectos como Organizations: Debating new agendas, Issues y perspectives; Grupo de discusión de estudios organizacionales, y Biblioteca virtual de estudios organizacionales. Asimismo, a partir del último año, se enfocó en aplicar el área de especialización teórica al estudio de la educación superior y, particularmente, a la universidad mediante proyectos de investigación como Re-conociendo a la universidad, sus transformaciones y su porvenir, el cual fue coordinado también por, además de Ibarra, por Luis Porter, animadores de la escritura del libro de la universidad imaginada.

El proyecto de reconocer a la universidad, que convocó a 74 investigadores de diez instituciones diferentes, partía de la premisa de que aquella había sufrido mutaciones en la última década del siglo XX, las cuales debían ser explicadas para comprender sus efectos futuros; es decir, se reconocía implícitamente que la universidad ya no era la misma y que, por tanto, era necesario diseñar todo un programa de investigación para volver a conocerla mediante el planteamiento de preguntas como estas:

¿Se puede considerar la transformación de la universidad en México como un proceso distinto e independiente de la transformación de la universidad en otros países del mundo, o ella obedece a una tendencia integral asociada a los procesos de mundialización que se sintetizan en la llamada "sociedad del conocimiento"?

¿Qué importancia tiene la universidad de hoy para la sociedad? ¿En qué medida ella seguirá siendo espacio para impulsar la construcción de una sociedad más justa mediante la socialización amplia e indiscriminada de conocimiento y cultura? ¿Hasta dónde la universidad puede favorecer el respeto de los derechos humanos, la procuración de la defensa social del medio ambiente y la protección de la diversidad y pluralidad que caracterizan la nación? 

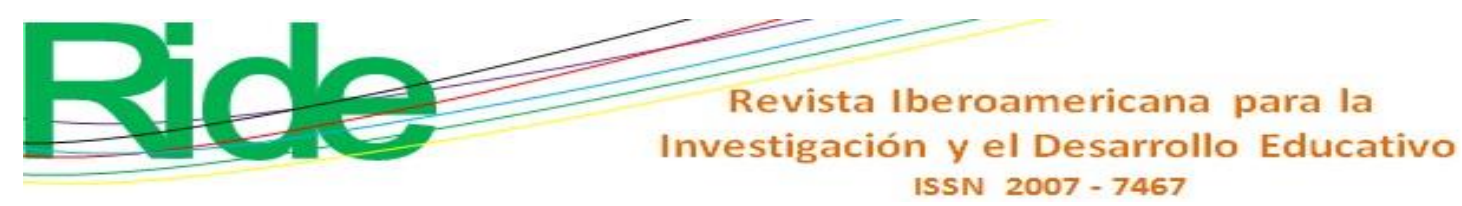

¿En qué sentido se han modificado las relaciones entre el Estado, la universidad y la sociedad? ¿Qué importancia adquieren tales relaciones a la luz de la vinculación del conocimiento con la economía y la política? ¿Cómo ha sido posible instrumentar específicos procedimientos de auditoría en las instituciones y defender a la vez su autonomía? O para plantearlo en otros términos, ¿Qué significado adquiere hoy la autonomía universitaria bajo los nuevos dispositivos de fiscalización y financiamiento a concurso? ¿Hasta dónde los problemas de la universidad se encuentran asociados a las estrecheces financieras que ha enfrentado históricamente y hasta dónde a un uso discrecional e inequitativo de los recursos disponibles?

¿En qué sentido debe la universidad transformar su estructura de gobierno y de toma de decisiones para posibilitar la producción de consensos, asegurando la participación de la comunidad? ¿Cuál es el equilibrio deseable entre las distintas fuerzas de la universidad que garantice gobernabilidad, es decir, preservación de la pluralidad y la diversidad, canalización de los conflictos y generación de consensos? ¿Cómo se puede construir una estructura de normas que sean compartidas, aceptadas y respetadas por todos y que proporcionen un escenario cierto en el que las reglas del juego acordadas conduzcan a relaciones más justas y equitativas?

¿Cuál es el lugar que ocupan y que deben ocupar los actores de la universidad en el nuevo escenario institucional? ¿De qué manera se han ido reconstituyendo las identidades de académicos, estudiantes, trabajadores y funcionarios, y en qué medida han resultado adecuados los mecanismos de conducción utilizados para orientar sus comportamientos? ¿Acaso es necesario transformar los mecanismos de conducción vigentes para fortalecer la participación de la comunidad universitaria atendiendo a su sentido esencial como colectividad?

¿Qué papel ha jugado la normatividad en los procesos de transformación de la universidad? ¿En qué medida se requieren modificaciones normativas para 

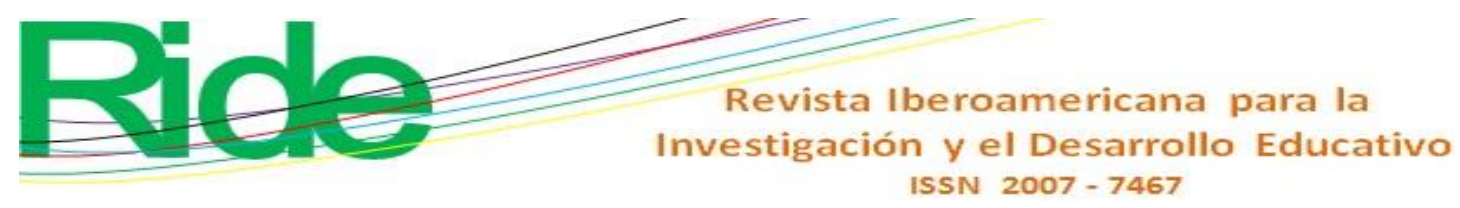

otorgar estabilidad y certidumbre a la universidad como espacio social de participación en torno a la producción, transmisión y socialización del conocimiento? ¿Hasta dónde la poca claridad normativa se ha constituido como fuente de conflictos y obstáculo de transformación de la universidad?

¿Qué tipo de universidad podremos tener en el futuro? ¿En qué medida es necesario reorientar tales tendencias y trabajar por un proyecto universitario distinto? ¿En qué aspectos debería ser distinta la universidad que hoy se perfila de la que pudiera garantizar a la sociedad su porvenir? ¿Cuáles son los ejes que podrían orientar un nuevo proyecto universitario que garantice su porvenir como institución cultural básica de la sociedad? (Ibarra, S/A, pp. 47-48).

En el Encuentro de Especialistas en Educación Superior, realizado en julio del 2000, fueron presentadas ponencias para responder a las preguntas planteadas, las que dieron origen a una obra colectiva titulada Re-conociendo a la universidad, sus transformaciones y su porvenir, la cual fue publicada en cinco tomos con los siguientes títulos: 1) Estado, universidad y sociedad, entre la globalización y la democratización; 2) Evaluación, financiamiento y gobierno de la universidad: el papel de las políticas; 3) Los actores de la universidad: ¿unidad en la diversidad?; 4) La universidad y sus modos de conocimiento: retos del porvenir, y 5) La transformación de la UNAM: 125 propuestas argumentadas.

En el 2001 se desarrolló otro proyecto bajo la coordinación, nuevamente, de Ibarra, Porter y Cazés, ahora para promover la primera etapa del Proyecto Interinstitucional de Autoestudio de las universidades públicas mexicanas, la cual procuraba reunir a un equipo de investigadores de las universidades públicas del país para profundizar en las transformaciones detectadas en el proyecto anterior; de este modo se intentaba incidir en los cambios futuros de las instituciones locales.

Los estudios elaborados por los investigadores de cada universidad fueron encausados en cuatro dimensiones: 1) reconocer las historias de la institución; es decir, rebasar la crónica oficial de la institución mediante la recuperación de las diferentes versiones históricas que emanaban de los sujetos que hacen a la universidad; 2) conocer, actualizar y comparar los “signos vitales" de la institución para elaborar un estudio cuantitativo que permitiera ofrecer 

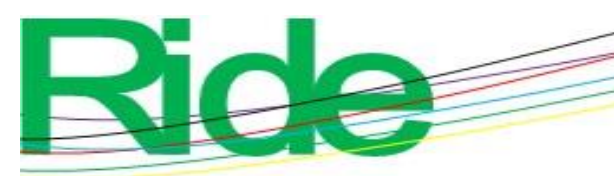

\section{Revista Iberoamericana para la \\ Investigación y el Desarrollo Educativo}

ISSN $2007-7467$

un diagnóstico específico; 3) ubicar a cada institución como parte constitutiva de una geografía universitaria nacional para reconocer que la especificidad institucional era parte de un sistema nacional de educación superior, y 4) reflexionar sobre la naturaleza y las prácticas cotidianas del trabajo universitario, de su contenido, resultados e impacto para saber cuál proyecto de vida construían los universitarios cotidianamente (Ibarra, S/A).

Como en los proyectos anteriores, los resultados pudieron observarse en actividades como la integración del equipo interinstitucional, la realización del Primer Encuentro de Auto-Estudio de las universidades públicas mexicanas en la UNAM y la publicación de una obra en dos tomos que constituyó el primer estudio global de las universidades de México; se trató de la Geografía política de las universidades públicas mexicanas: claroscuros de su diversidad en 2003 y 2004.

Posteriormente, entre 2004 y 2006, Ibarra, Porter y Cazés —a los que se agregaron Axel Didriksson y José Gandarilla- promovieron la segunda etapa del Proyecto Interinstitucional de Autoestudio de las universidades públicas mexicanas, en el que participaron 64 investigadores de 25 instituciones distintas, los cuales desarrollaron análisis particulares sobre sus propias universidades con base en las siguientes cinco temáticas generales: con el primer tema se buscaba conocer cuál era la situación de la autonomía universitaria, para lo cual se podían abordar aspectos como el jurídico, académico, financiero, estructura de gobierno, transformación legislativa y recomendaciones respecto al tema.

El segundo tema se centró en el financiamiento, que sería estudiado con base en la premisa de que el neoliberalismo había provocado cambios en la forma de asignar los recursos, por lo que se recomendaba enfatizarse en los montos de los recursos y la procedencia estatal, federal o de otro tipo, la trayectoria, las políticas y programas locales, así como las perspectivas para cada universidad.

El tercer tema se enfocó en apreciar los cambios en la normatividad que regula la gobernabilidad universitaria con estudios específicos vinculados con la comparación institucional antes y después de las modificaciones, el impacto de los cambios, la participación de la comunidad en los órganos de gobierno, el funcionamiento de las juntas u 


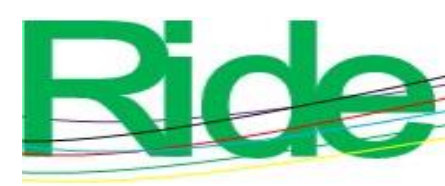

Revista lberoamericana para la

Investigación y el Desarrollo Educativo

ISSN $2007-7467$

otras formas de gobierno central, el funcionamiento de los órganos colegiados y los conflictos universitarios recientes.

El cuarto tema se orientó hacia la evaluación y la remuneración del trabajo académico, puesto que no se tenían estudios sobre los efectos de los programas de remuneración a concurso impuestos en las últimas décadas. La convocatoria invitaba a los investigadores a reconocer la modalidad local y sus efectos al considerar variables como la estructura organizativa del modelo académico-laboral y las características del personal académico, los programas de deshomologación salarial aplicados y sus consecuencias, así como una valoración general para hacer propuestas.

La última línea de trabajo se justificó con la reivindicación de lo imaginario como conocimiento necesario en la universidad, por lo que se les hizo a los investigadores el siguiente exhorto:

Reflexionar sobre el papel que juegan tanto en la universidad como en nuestra forma de conocer las expresiones del arte, la forma literaria y poética, pero también las artes visuales, la imagen y el sonido, como lenguajes que son invisibles para formas tradicionales de conocer que ocultan o disuelven los caracteres existenciales, subjetivos, afectivos, del ser humano. Se convoca igualmente a discutir sobre formas de conocer y de expresar lo conocido, que nos permitan ver con nuevos ojos las relaciones del ser humano en la universidad, con el prójimo, con la sociedad, con el mundo (Ibarra, S/A, p. 43).

El encuentro de autoestudio arrojó como resultado en 2007 la publicación del libro Disputas por la universidad: cuestiones críticas para confrontar su futuro, por el Centro de Investigaciones Interdisciplinarias en Ciencias y Humanidades de la UNAM. Con esta obra se divulgaron los cambios recientes en las universidades públicas del centro y de las entidades, lo que se convirtió en un diagnóstico global sobre las transformaciones de la universidad mexicana a inicios del siglo XXI. Esto sirvió como base para formular el siguiente proyecto de investigación colectiva, titulado Universidad 2030: escenarios de futuro, desarrollado durante los años 2007-2009 en dos fases. 

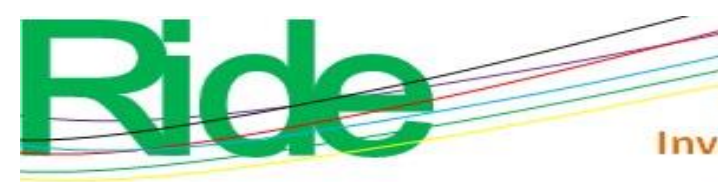

Revista Iberoamericana para la

Investigación y el Desarrollo Educativo

ISSN $2007-7467$

La primera estuvo constituida por la argumentación del proyecto en torno a dos ideas: una, que un análisis serio de las transformaciones de la universidad solo se podía hacer desde el contexto de la sociedad y, otra, que el nuevo estudio se realizaría bajo la perspectiva de la disciplina reciente relacionada con la construcción de escenarios futuros; en esta fase también se diseñó una página de internet para difundir la convocatoria, se conformó el comité interinstitucional y se realizó un seminario preparatorio titulado Imaginación rigurosa: una metodología para visualizar escenarios a futuro de la universidad (Ibarra, S/A, p. 38).

La segunda fase fue su conversión en el Proyecto interinstitucional de autoestudio de las universidades públicas mexicanas. Etapa III: la universidad en México en el año 2030: imaginando futuros, a cargo del trío de académicos que había impulsado los estudios sobre la universidad: Daniel Cazés, Eduardo Ibarra y Luis Porter. La justificación de este trabajo se sustentó en un diagnóstico de la universidad mexicana como institución inmersa en la "sociedad del conocimiento", la cual se presentó de manera cuestionada por su "condición posmoderna" que le demanda atender exigencias particulares con distintas formas de enseñanza y complejas maneras de organización, requerimientos que la obligaban a "transformaciones institucionales fundamentales, entre las que se encuentran la diversificación y reorganización de los sistemas de educación superior y de ciencia y tecnología, la transformaciones de las estructuras y funciones de la universidad y el fin del monopolio universitario sobre el conocimiento" (Ibarra, S/A, p. 35).

Al respecto, cabe resaltar que la universidad ha sido concebida tradicionalmente como la institución encargada de generar y distribuir el conocimiento, aunque con el advenimiento de la sociedad de la información y el conocimiento, su estructura y papel han cambiado; ahora debe competir en el desarrollo de una de sus actividades sustantivas con otras instituciones, como las empresas, las agencias gubernamentales o las "instituciones privadas nacionales y extranjeras en los mercados de servicios educativos, la producción científica y la innovación tecnológica" (Ibarra, S/A, p. 35).

Con base en la interrogante acerca de qué tipo de universidad tendremos para el futuro, el comité convocó a los investigadores para que imaginaran las transformaciones que debería tener la universidad para asegurar la producción, transmisión y apropiación del 

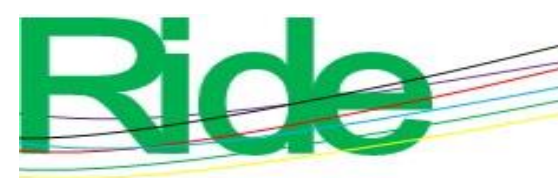

Revista Iberoamericana para la

Investigación y el Desarrollo Educativo

ISSN 2007 - 7467

conocimiento en un modelo de mercado desinteresado de las necesidades sociales; la producción del conocimiento imaginado, en consecuencia, se debería materializar sobre los siguientes ejes:

La sociedad del conocimiento y el llamado mundo global: México y su Universidad en el año 2030; el futuro de la oferta, la demanda y las modalidades educativas en México; los nuevos modos de coordinación y regulación de los sistemas de educación superior y de ciencia y tecnología: ¿qué educación y qué ciencia para el 2030?; las nuevas formas de producción del conocimiento y la reorganización de la universidad: flexibilidad, redes y ciberuniversidad?; el financiamiento, la comercialización y los mercados del conocimiento en el 2030: ¿será posible el capitalismo académico en México?; las formas de gobierno y la gobernabilidad de la universidad en el siglo XXI: imaginando la participación, la eficacia y la legitimidad de la nueva universidad; el futuro de la gestión universitaria: profesionalización de la dirección universitaria y sistema flexible de gestión en redes; la carrera académica del futuro: diversificación de la profesión académica, nuevas funciones y nuevas identidades; los estudiantes en el 2030: ¿ciudadanos de conocimiento?: composición del cuerpo estudiantil, modalidades de enseñanza y papel de las nuevas tecnologías (Ibarra, S/A, pp. 35-36).

De los trabajos de investigación que los integrantes del grupo interinstitucional presentaron en el seminario respectivo resultó - como ya era común en los proyectos del grupo de Ibarra - el libro Las universidades públicas mexicanas en el año 2030: examinando presentes, imaginando futuros, publicado conjuntamente por la UNAM y la UAM. Con este producto académico se rebasó el diagnóstico de la historia de la universidad mexicana y se pasó al ejercicio sistemático de la imaginación para generar propuestas a la inercia que las transformaciones del Estado y la economía le impusieron a la educación superior.

Aunado a esto, se empezó a estructurar el Laboratorio de Análisis Institucional del sistema Universitario Mexicano (LAISUM) con el propósito de proporcionar información sobre las universidades a través de un portal de internet. El laboratorio funcionaría con tres 


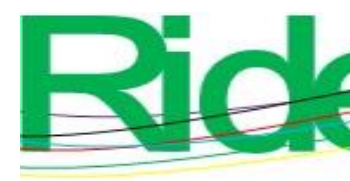

Revista lberoamericana para la

Investigación y el Desarrollo Educativo

ISSN 2007 - 7467

secciones: la primera con el propósito de recopilar notas periodísticas para reconocer la orientación de la opinión pública respecto a las coyunturas; la segunda para ofrecer una geografía política con información significativa de las universidades, y la tercera con un espacio virtual para reflexionar sobre la universidad a partir de marzo de 2011 (Ibarra, S/A).

El último trabajo que Ibarra impulsó buscaba hacer un balance de 20 años de modernización educativa en el nivel superior con el proyecto Efectos de las políticas gubernamentales en las universidades públicas mexicanas (1989-2009), el cual se desarrollaría entre 2011 y 2014 bajo su dirección. Este procuraba presentar una comparación institucional de seis casos de universidades públicas para apreciar la diversidad del sistema universitario mexicano. Esperaba realizar la fase IV del encuentro de autoestudio de las universidades, elaborar un almanaque con información de las 55 universidades que integraban el sistema universitario mexicano y publicar los resultados; sin embargo, lo sorprendió la muerte (Rodríguez, 2013).

\section{Síntesis del diagnóstico histórico en el libro de la universidad imaginada}

La perseverancia en el estudio de la educación superior por parte de Ibarra, Porter y Cazés llevó a los dos primeros a impulsar la escritura de un libro en el que se planteaba una especie de utopía de universidad futura con base en el conocimiento de lo que la educación superior mexicana había sido en el pasado; esto desde una postura crítica sobre su funcionamiento entre finales del siglo XX y principios del XXI. Específicamente, El libro de la universidad imaginada. Hacia una universidad situada entre el buen lugar y ningún lugar nació durante el seminario Imaginación rigurosa: una metodología para visualizar escenarios futuros de la universidad, conducido por Riel Miller como preparación para el encuentro Universidad 2030: escenarios de futuro.

Ahora bien, independientemente de la forma novedosa en que fue producido y presentado, en este momento interesa resaltar el balance crítico del que parten los autores para sustentar sus propuestas. Por ejemplo, sobresale la postura reflexiva de Eduardo Ibarra ante el discurso de la "excelencia", el cual —según dicho autor - fue introyectado 


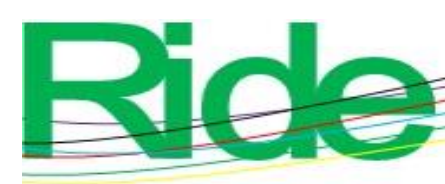

Revista Iberoamericana para la

Investigación y el Desarrollo Educativo

ISSN 2007 - 7467

socialmente mediante la "maquinaria del poder de la que formamos parte todos nosotros". Por eso, para Ibarra ese discurso lleno de datos y documentos que procuraba demostrar una sólida argumentación en realidad ocultaba "las mediocridades de esos malos académicos que, como fantasmas a la caza de las bolsas de dinero bajo concurso, deambulan alegres en nuestras universidades de hoy, sin importarles demasiado las aspiraciones más plenas del compartir, aprender, reflexionar y crear" (Ibarra et al., 2012, p. 13); aun así, también vale comentar que Ibarra et al. (2012) reconocieron la existencia de notables académicos que se ajustaban a las aspiraciones descritas, a pesar de "esa corrupción sistémica de la universidad, que es ya moneda de curso legal" (Ibarra et al., 2012, p. 13).

Esta caracterización de los buenos y malos académicos como resultado de la política de la "excelencia" educativa se apoya en un texto de Luis Porter, titulado El alma del académico bajo el posmodernismo, en el cual el referido autor denuncia lo siguiente:

[El académico] vende su talento y su tiempo para trabajar en la investigación o en la docencia con la misma actitud que lo hace un obrero de una fábrica, sometido y forzado a aceptar condiciones que deberían ser inaceptables, olvidando o desconociendo las necesidades nacidas de su propia vocación, sensibilidad y compromiso (Porter citado por Ibarra et al., 2012, p. 13).

El segundo aspecto recurrente del diagnóstico histórico del que nace la utopía imaginada es el de la colonialidad epistémica o colonización del saber, la cual, según los autores, "ha impuesto el silencio a nuestras culturas ancestrales, y con ellas a nosotros mismos, mediante la verdad totalitaria de la razón occidental” (Ibarra et al., 2012, p. 72). Para apoyar esta postura, los autores recurren a la idea de que la conquista europea también trajo a la universidad "para enseñarles a los nativos cómo hablar, cómo nombrar las cosas, cómo pensar y cómo pensarse a sí mismos, a la par de la ciencia moderna, la de los conquistadores y sus valores, junto con sus creencias y formas de vida" (Ibarra et al., 2012, p. 72). 

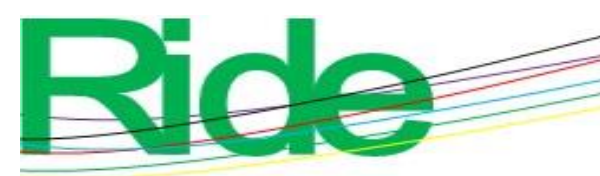

\section{Revista Iberoamericana para la Investigación y el Desarrollo Educativo ISSN 2007 - 7467}

No obstante, a pesar de que la universidad colonial fue reformada, el conocimiento moderno niega la identidad de los otros que sobreviven y han surgido de las transformaciones sociales para imponer unas formas de conocimiento sobre otras con que se refuerza la condición colonizada.

En este tema aparece el discurso de una de las participantes en la escritura del libro: Lourdes C. Pacheco, quien en su texto La universidad de la incompletud (s. f.), también incluido como recuadro de apoyo, especifica la forma textual en que la ciencia impone y desconoce al mismo tiempo otros documentos que no se apegan al canon científico, y junto con ello a los sujetos que los portan como indígenas, mujeres y poetas. Luego de narrar el proceso de invasión de la racionalidad científica y tecnológica en todos los ámbitos de la vida, Pacheco sostiene:

Las universidades se han convertido en universidades de la incompletud al suprimir, inimaginar, desacreditar, olvidar, subordinar, expulsar otros conocimientos (otras formas de conocer y otros lenguajes) (De Souza, 2004). Impide la premisa de la completud del conocimiento como totalidad. Porque conocer es un proceso de la materia viva (Bagú, 2003) donde todo el corpus participa en ella de manera involuntaria (sin pasar por la conciencia) (Ibarra et al., 2012, p. 74).

La autora citada sintetiza la historia de la universidad latinoamericana al señalar que su presencia ha sido catastrófica porque al privilegiar la lectura de la teoría europea y negar la posibilidad de un pensamiento propio, no se crearon proposiciones capaces de transformar la realidad. De hecho, aunque el 68 estudiantil cuestionó a esa universidad occidentalizada, las políticas derivadas, así como la apertura de la universidad a la sociedad (visibles en la masificación) y la relación con empresarios locales y el extensionismo fueron solo una mascarada, pues finalmente "las funciones de la universidad siguen siendo la investigación y la docencia: el lugar del pensamiento racional, el sujeto de la cultura central" (Ibarra et al., 2012, p. 74). 

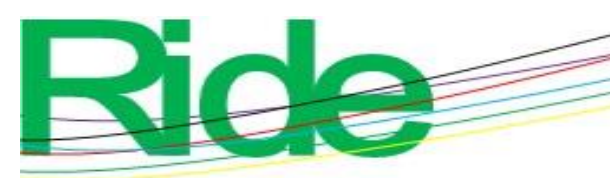

\section{Revista Iberoamericana para la Investigación y el Desarrollo Educativo ISSN $2007-7467$}

Los autores, en otras palabras, comparten la idea de que la educación universitaria del presente está en crisis debido a que emerge de teorías y formas institucionales que subordinan y excluyen, a pesar de supuestas reformas que buscan adecuarla a los tiempos. Al respecto, fundamentan su hipótesis en Pablo Latapí, quien en un discurso de 2007 externó cuatro preocupaciones a la UAM en el marco de su doctorado honoris causa.

Las preocupaciones en conjunto diagnostican el funcionamiento de la universidad a comienzos del siglo XXI en el siguiente sentido: en primer lugar, expresa su pesar por el discurso de la excelencia porque en la práctica educativa este promueve la arrogancia, el narcisismo y el egoísmo en los universitarios en formación; en segundo lugar, le preocupa la definición de calidad de la educación, ya que en la universidad se confunde solo con el aprendizaje de conocimientos, por lo que se aplica sin considerar la diferencia de los contextos donde se ubican las universidades; además, le preocupa que se inculque a los estudiantes las ideas de conseguir el escalafón más alto con el mejor salario para adquirir más pertenencias; esto es, volverlo materialista y consumista.

La tercera preocupación de Latapí es la idea de que toda universidad debe inscribirse en la llamada sociedad del conocimiento debido a que el planteamiento es incompleto al vincular su producción (actividad central de la universidad) solo a las necesidades económicas empresariales. En tal sentido, el referido autor asegura que las sociedades necesitan otros conocimientos derivados de las ciencias humanas; por ende, explica: 'Decimos 'sí' a la sociedad del conocimiento que incluya la universalidad de los saberes humanos, y advertimos contra la trampa de convertir a las universidades en fábricas de inventos prácticos; ellas son creaciones del homo sapiens, no las reduzcamos a talleres del homo faber" (Ibarra et al., 2012, p. 90).

Finalmente, la cuarta preocupación de Latapí tiene que ver con el predominio del conocimiento racional en las universidades, lo que califica como una situación equivocada y contradictoria, pues la educación va más allá del territorio de la razón. Es cierto que dicho autor no niega el avance de la ciencia y la importancia de sus aplicaciones tecnológicas, aunque sugiere no privilegiarlo como el único para abrir la universidad a los "ámbitos poco 


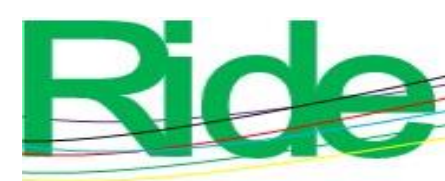

Revista Iberoamericana para la

Investigación y el Desarrollo Educativo

ISSN $2007-7467$

explorados pero esenciales del desarrollo humano que rebasan lo racional" (Ibarra et al., 2012, p. 91).

En definitiva, el diagnóstico de Ibarra et al. (2012) sirve de sustento para indicar que la universidad mexicana es "de piedra", porque la vida está ausente de ella y el conocimiento está petrificado en lo racional y separado hasta el infinito en las especializaciones, lo que contradice las múltiples capacidades con las que el humano enfrenta los problemas y desafíos de la complejidad real. Esto mutila no solo la formación de los estudiantes, sino también la de los maestros, cuyo comportamiento especializado los muestra "incapaces de responder a la concurrencia, las asociaciones, los antagonismos y las incertidumbres que ocurren en la vida, y a las formas múltiples e inciertas del conocimiento y la creación humanos" (Ibarra et al., 2012, p. 100).

Según los autores, la universidad mexicana también se encuentra desinstitucionalizada porque su funcionamiento deriva de las políticas estatales que provienen de los organismos internacionales y de la fuerza de la globalización. Así, al no construir un proyecto de reformas que valore su propio proceso histórico, se apega a la estandarización y con ello tergiversa, por ejemplo, el trabajo del académico que debe dedicarse a cumplir con trámites y papeleos para demostrar que cumple el rol asignado externamente.

Ante el hecho de que el conocimiento pertinente para la vida social se produzca en los centros de trabajo y en otros espacios de la vida colectiva (y no en las aulas, laboratorios y cubículos universitarios), hace de la universidad una institución innecesaria para la sociedad excluida y las comunidades subalternas; además, cuando se les incluye como en los periodos de masificación, se les despoja de su identidad comunitaria y se les obliga a aprender el conocimiento científico "para comenzar a ser como no son, para emular a su conquistador". En otras palabras, la universidad no solo es innecesaria, vieja y susceptible de acabar por razones como las siguientes: 


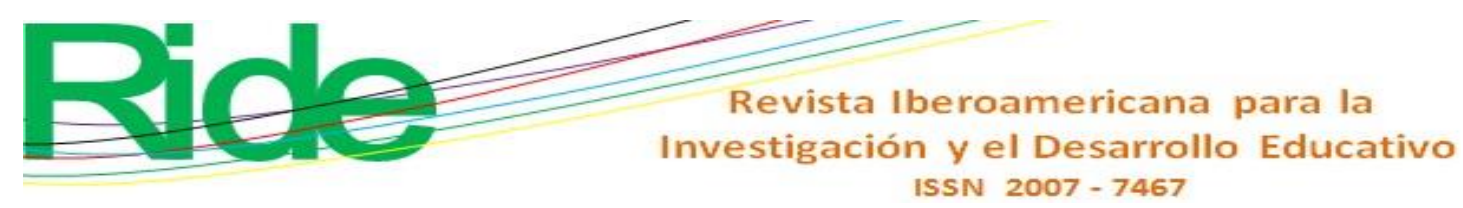

Son muchas las razones que nos hacen desear que esta vieja universidad, cada día más desfigurada e irreconocible, desaparezca, y junto con ella su banalización, sus falsos rituales, sus discursos mentirosos, sus recursos de poder basados en la arrogancia oculta bajo conceptos adulterados de rigor y método, de calidad y de excelencia, términos que en nuestro contexto no son más que la expresión de una retórica, tan hipócrita como falsa, que intenta encubrir la universidad utilitaria, esa cuyos proyectos, lejos de ser colectivizados, son apropiados de manera privada (Ibarra et al., 2012, p. 103).

Finalmente, la vieja universidad que los autores proponen superar con la universidad imaginada se caracteriza por fundamentar sus funciones en buscar "la formación del mal llamado 'capital humano"'; someterse al poder, por ejemplo, de las corporaciones comerciales hoy en día; culpar al individuo cuando no alcanza la instrucción que la universidad le proporción; adoptar la simulación y la corrupción como camino al éxito mediante premios a grupos de interés; eliminar el placer del conocimiento y la creatividad a través de la competitividad y el descrédito del prójimo; negar al sujeto y su subjetividad de estudiantes y maestros mediante la rigidez del currículo, y establecer una enorme distancia entre la petrificación de la universidad y la complejidad de la vida (Ibarra et al., 2012, p. 111).

\section{Conclusiones}

Para presentar un diagnóstico histórico de instituciones públicas como las universidades es importante tener claridad sobre los modos como se va tejiendo el acontecer humano en ellas y en el contexto en el que se construyen y funcionan; es decir, es necesario tener una idea de las teorías de la disciplina histórica para comprender los modos de articulación entre el presente y el pasado, el pasado y el presente, y el presente y el futuro, pues solo de esa manera es posible no solo comprender el tiempo que se vive, sino imaginar $\mathrm{y}$ vislumbrar un porvenir. 

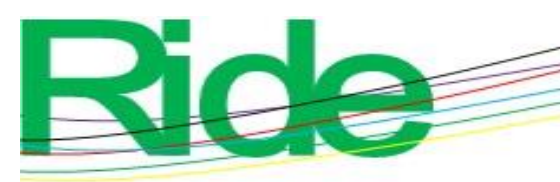

Revista Iberoamericana para la

Investigación y el Desarrollo Educativo

ISSN $2007-7467$

Igualmente, además de una epistemología de la ciencia en general y de la ciencia histórica en particular, se deben conocer las teorías surgidas de nuevas áreas de creación de conocimiento como los estudios institucionales y organizacionales para generar un proceso que revele conocimiento profundo sobre el hecho que se estudia.

Sin embargo, este análisis hermenéutico del libro de la universidad imaginada ha enseñado que los sujetos que estudian a dicha institución —además de construir un marco teórico y metodológico desde una disciplina científica y crear instituciones para aplicar esa teoría a nuevos estudios - la van forjando con sus actos de vida y sus reflexiones permanentes sobre su desempeño general en su contexto. Aunado a ello, el conocimiento que logran es más profundo y sirve de apoyo para imaginar otras formas de funcionamiento a la luz de un ideal que concomitantemente se perfila.

Ese fue el ejemplo de vida de Eduardo Ibarra Colado, quien durante tres décadas vivió un proceso en el que simultáneamente conoció y desarrolló los estudios dedicados a conocer y explicar las instituciones y las organizaciones a través de la docencia, la dirección de tesis y la investigación, y aplicó la teoría organizacional a la educación superior para luego, conforme avanzaba en sus propósitos científicos, delimitar su objeto de estudio al conocimiento de la universidad mexicana, lo que le permitió conformar una categoría llamada sistema universitario mexicano.

Además de ello, sobresale en la vida de Ibarra su confianza en la importancia de la colectividad para la construcción de conocimiento, lo que demostró con el impulso de varios proyectos de investigación y la publicación de los resultados sobre la educación superior y la universidad, los cuales manifiestan conciencia del presente y búsqueda de explicaciones en el pasado mexicano y del mundo. Asimismo, la respuesta de otros investigadores de América y Europa a las convocatorias para estudiar a la universidad le dio solidez a la interpretación colectiva del pasado y del presente de la universidad y promovió la formación de otros investigadores locales para fomentar el autoconocimiento de esas casas de estudio.

La secuencia cronológica y temática de los proyectos de investigación sobre la educación superior y la universidad —impulsados primero por Ibarra y luego por Cazés y Porter - revelan la construcción de un diagnóstico histórico de la universidad mexicana, pues 


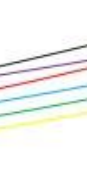

Revista Iberoamericana para la

Investigación y el Desarrollo Educativo

ISSN 2007 - 7467

comienzan por pensar y afinar la metodología institucional, luego ponen énfasis en entender el impacto de las políticas y discursos internacionales de fines del siglo XX y principios del XXI, así como su adopción nacional en el conjunto de universidades mexicanas con una visión global para construir con fundamentos escenarios futuros en un sentido crítico y alternativo, los cuales no se abordan en este texto, pues la interpretación de la utopía que imaginan da para otro artículo.

En definitiva, el diagnóstico — como era de esperarse después de tan largo proceso de estudio - es radicalmente crítico de los sujetos que con sus prácticas hacen a la universidad y originan las rutinas institucionales. Las calificaciones de universidad en crisis, incompleta, colonizada, de piedra, desinstitucionalizada, sometida y vieja obligan a revisar los argumentos y llevan a coincidir en la necesidad de erigir una nueva universidad o de formular una utopía que le dé un nuevo sentido ético.

\section{Referencias}

Buendía, A. (2014). Laberintos y enjuegos: (re) encuentros con Eduardo Ibarra Colado. Recuperado de http://publicaciones.anuies.mx/books/204/laberintos-y-enjuegos-reencuentros-con-eduardo-ibarra-colado

Gadamer, H. (1975). Verdad y método. Salamanca: Sígueme.

Ibarra, E. (S/A). Curriculum vitae. Recuperado de http://laisumedu.org/DESIN_Ibarra/ibarra/pdf/cv-eic-2013-esp.pdf.

Ibarra, E., Porter, L., Álvarez, L. et al (2012). El libro de la universidad imaginada. Hacia una universidad situada entre el buen lugar y ningún lugar. México: UAMCuajimalpa/Juan Pablos Editor.

Pacheco, L. (s. f.). La universidad de la incompletud. Recuperado de http://www.laisumedu.org/DESIN Ibarra/autoestudio2004/docs2005/Pacheco.pdf. 
Porter, L., En memoria de Eduardo Ibarra Colado (1957-2013). Recuperado de http://www.revistaraes.net/revistas/raes6 mem1.pdf

Porter, L. (2014). Conversaciones con Eduardo Ibarra Colado. Recuperado de file://C:/Users/USUARIO/Downloads/art\%C3\%ADculo redalyc 457645126005.pdf

Rodríguez, R. (2013). Eduardo Ibarra Colado. Recuperado de http://campusmilenio.mx/index.php?option=com_k2\&view=item\&id=144:eduardoibarra-colado\&Itemid $=140$

Sin autor (s. f.). Publicaciones del Dr. Eduardo Ibarra Colado. Recuperado de https://www.ses.unam.mx/integrantes/uploadfile/eibarra/Publicaciones.pdf

Sin autor (s. f.). Resumen curricular. Recuperado de http://www.laisumedu.org/DESIN Ibarra/ibarra/perfil.htm 